\title{
De la solución fenomenológica a la paradoja de McTaggart, al problema del «pluralismo» en la descripción de la realidad temporal
}

\section{From the phenomenological solution to McTaggart's paradox to the problem of pluralism in the description of temporal reality}

\author{
LIC. CLAUDIO CORMICK \\ (UBA-Paris VIII/CONICET)
}

Recibido: 29-XII-2013 Aprobado: 25-II-2014

\begin{abstract}
RESUMEN
Intentaremos continuar en este trabajo la propuesta de una solución a la paradoja de McTaggart a partir de la retoma de una línea de análisis que se remonta a Dummett, y su complementación con ciertas tesis de la fenomenología merleaupontyana. Para ello tendremos que profundizar las posiciones del autor de Truth and other enigmas en el sentido de la objeción contra una «descripción completa» de la realidad y que favorecen, por el contrario, de una pluralidad de «descripciones máximas». Nuestro intento no debe, sin embargo, adoptar un «pluralismo» que se exponga a las objeciones de Nicholas Smith, a quien replicaremos a su vez apelando a la noción fenomenológica de «síntesis de transición». Concluiremos que el «pluralismo» de descripciones temporales que nos permite escapar a la paradoja de McTaggart no resulta incompatible con el requisito de «componibilidad» planteado por Smith.
\end{abstract}

PALABRAS CLAVE

TIME, MERLEAU-PONTY, MCTAGGART, DUMMETT, SMITH

\begin{abstract}
(C) Contrastes. Revista Internacional de Filosofía, vol. XX-Nº1 (2015), pp. 7-26. ISSN: 1136-4076

Departamento de Filosofía, Universidad de Málaga, Facultad de Filosofía y Letras Campus de Teatinos, E-29071 Málaga (España)
\end{abstract}


In this paper we attempt to continue the proposal of a solution to McTaggart's paradox on the basis of a return to a line of analysis traceable back to Dummett, and its implementation with certain tenets of Merleau-Pontyan phenomenology. With this aim, we have to deepen the positions of the author of Truth and other enigmas which point towards an objection against one "complete description" of reality and favour, on the contrary, a plurality of "maximal descriptions". Our attempt must not, however, adopt a "pluralism" which can be targeted by Nicholas Smith's objections, to whom, in turn, we reply by turning to the phenomenological notion of "synthesis of transition". We conclude that the "pluralism" of temporal descriptions that allows us to escape from McTaggart's paradox is not incompatible with the requisite of "compossibility" laid out by Smith.

KEYWORDS

TIME, MERLEAU-PONTY, MCTAGGART, DUMMETT, SMITH

\section{INTRODUCCIÓN}

INTENTAREMOS EN ESTE TRABAJO continuar el cruce, iniciado en artículos previos de nuestra autoría, entre la fenomenología merleaupontyana del tiempo y los debates contemporáneos en la teoría del tiempo desarrollada en el marco de la filosofía anglosajona, y cuyo hilo conductor central es el debate entre teorías «A»y «B». Esquematizando nuestro desarrollo:

- Mientras en otros trabajos -cuyos resultados resumiremos en la sección (I) de este artículo- hemos mostrado de qué modo las críticas de Merleau-Ponty a la filosofía trascendental podían extrapolarse en dirección de una solución de la paradoja de McTaggart, en el presente artículo intentaremos enfrentar una crítica que ya presupone el paso por una solución del tenor de la que hemos propuesto.

- Por ello, en la sección (II) nos acercaremos a esta discusión explicitando una consecuencia polémica del acercamiento «situacional» merleaupontyano: la tesis de que el tiempo debe describirse desde una perspectiva situada en él parece llevarnos a una segunda tesis, sobre la multiplicidad de descripciones de la realidad temporal, posición con la cual, por lo demás, el enfoque merleaupontyano parecería confluir con las reflexiones de Michael Dummett.

- Sobre esta base es que finalmente, en la sección (III), nos referiremos a una crítica formulada por Nicholas Smith en un trabajo del año 2011, la cual replantea la paradoja de McTaggart no señalando la presunta inconsistencia que simpliciter involucrarían las determinaciones de presente, pasado y futuro, sino atacando, de manera más específica, la forma en que el abordaje de la realidad con estas «determinaciones A» pretendería escapar a la inconsistencia a través de mantener separadas las distintas descripciones de la realidad temporal. Responderemos a esta crítica por la vía de, a la vez, conceder la plausibilidad de la exigencia de Smith de volver desde el «pluralismo» temporal a algún tipo de descripción unificada, pero, por otra parte, defender la tesis de que el abordaje 
merleaupontyano consigue efectivamente aunar el «momento» situacionalpluralista del análisis -clave para superar la presunta inconsistencia de las «determinaciones $A$ »- con un acercamiento al tiempo como flujo unificado.

\section{UNA SOLUCIÓN SITUACIONAL MERLEAUPONTYANA \\ A LA PARADOJA DE MCTAGgaRT}

Reconstruyamos brevemente algunos problemas básicos. Ante todo, recapitulemos algunos puntos sobre el «lado» fenomenológico de nuestro cruce. Podemos sintetizar las tesis de Merleau-Ponty sobre el tiempo de la siguiente manera:

1. Ante todo, el abordaje merleaupontyano sobre la temporalidad tiene un carácter situacional. Como señalamos en trabajos previos, es posible trazar una continuidad entre una obra temprana como la Fenomenología de la percepción y textos como las notas de trabajo de Lo visible y lo invisible en la medida en que, en ambos casos, encontramos una crítica a lo que Merleau-Ponty llama un «sobrevuelo» del tiempo: solo hay tiempo -insiste el fenomenólogo desde 1945 hasta 1960- para una subjetividad situada, que lo experimenta «desde adentro» de él, que tiene un punto de vista en el tiempo; el intento de la filosofía trascendental «intelectualista» de constituir el tiempo bajo la mirada de una conciencia no situada lleva a despojarlo, sin proponérselo, de las determinaciones de presente, pasado y futuro ${ }^{1}$.

2. Por otro lado, en ambos trabajos merleaupontyanos encontramos que el aspecto «situacional» de su tratamiento, que el filósofo enfatiza para delimitarse de las filosofías «intelectualistas», está en tensión con una igual crítica al empirismo. Mientras la tematización de una subjetividad «desembarazada» de un punto de vista en el tiempo adolece, según vimos, del defecto de «nivelar» el tiempo, desfigurándolo, este acercamiento intelectualista tiene no obstante la legitimidad parcial de buscar dar cuenta de la unidad del tiempo, de nuestra no-cerrazón en una perspectiva puntual. El empirismo, de forma simétrica al intelectualismo, nos brinda en efecto una tematización de nuestro carácter de situados, pero lo hace de modo tal que nos priva de la síntesis que asegure, a lo

1 Este argumento es desarrollado en más detalle en nuestro Cormick (2014). El apoyo textual más importante de nuestras tesis en la obra merleaupontyana se encuentra en MerleauPonty (1985): 419; 420; 423 y Merleau-Ponty (2010): 165; 170-171; 215. 
largo del desplazamiento de un presente por otro, que efectivamente sigamos teniendo «el tiempo», y no «tiempos» ${ }^{2}$.

Habiendo recuperado así el carácter situacional como su elemento distintivo, hemos podido establecer un -creemos- fructífero cruce entre el tratamiento merleaupontyano del tiempo y un problema proveniente de otra tradición, pero al cual pueden aplicarse las críticas de Merleau-Ponty a la filosofía trascendental. Nos referimos con esto a la llamada «paradoja de McTaggart», la cual puede verse como el resultado de una exploración, entre otras, de qué carácter nos presenta la temporalidad cuando no partimos de describirla desde un punto de vista situado en el presente. Para McTaggart las tres determinaciones de presente, pasado y futuro -las determinaciones propias de la que llama «serie A»-se caracterizan por el hecho de poder atribuirse a los momentos de forma variable. Mientras las relaciones -propias de la llamada «serie B»- de anterioridad y posterioridad caracterizan de manera invariable a un par de eventos -puesto que si uno de ellos ha sido alguna vez anterior a otro, siempre lo será, independientemente de si ellos son presentes, pasados o futuros-, las determinaciones de la «serie A» deben, precisamente para que exista un paso del tiempo, modificarse ( $c f r$. McTaggart, 1927: 10). No se puede decir de un momento que sea simplemente -por ejemplo-futuro, puesto que lo es solo desde el punto de vista de algún momento en particular, pero desde otros será presente o pasado. McTaggart insiste: si obtenemos aquí determinaciones unívocas -decir, en nuestro ejemplo, que un momento es futuro, y no atribuirle otro carácter temporal- será según una relación con algún otro momento en el tiempo, pero precisamente este otro momento cualquiera desde el cual las atribuimos, el cual sirve de punto de referencia, nos presenta otra vez el problema inicial: él ha de ser, según desde dónde lo consideremos, tanto presente como pasado o futuro. Y así indefinidamente (cfr. ibíd.: 21. Cfr. también la formulación original en McTaggart (1908): 468). No podemos atribuir a ningún momento ninguna de las tres determinaciones de la «serie A» con carácter absoluto -puesto que debe tener, en principio, todas ellas, o de lo contrario no habría paso del tiempo-, y si le atribuimos una u otra con carácter relativo a otro momento del tiempo, el mismo problema se nos presenta respecto a este otro momento, que es homogéneo al primero. En conclusión, la que McTaggart denomina «serie A» aparece como afectada de una contradictoriedad de la que solo podemos escapar mediante una regresión al infinito de puntos de referencia.

No nos extenderemos aquí sobre de qué modo es posible «instrumentalizar» una filosofía del tiempo de corte fenomenológico para responder a este

2 La contraposición entre «tiempo» y «tiempos» aparece en Merleau-Ponty (2010): 170-171, en un pasaje paralelo a lo que podemos encontrar -a partir de la necesidad de superar la fragmentación de los presentes-, en (1985): 422 y 428-430. 
enigma de su contraparte analítica. Resumiendo, digamos únicamente que, partiendo del principio de que los caracteres de pasado y futuro presuponen que exista una subjetividad situada en el presente, entonces la atribución de las «determinaciones A» no sigue la dirección que cree McTaggart, sino la opuesta. Esto es: no se trata de que tengamos momentos sobredeterminados con caracteres incompatibles a menos que especifiquemos con relación a qué otro momento es que poseerían una determinación u otra -y así al infinito-, sino que, a la inversa, necesitamos partir de tener algún punto de referencia, algún momento que cuente como «punto cero», como presente, puesto que de lo contrario no habrá sobredeterminación sino indeterminación con relación a los tres caracteres pertinentes (que es, mutatis mutandis, la crítica que MerleauPonty hacía a la filosofía de tipo trascendental). La perspectiva del presente es la que funda el sistema mismo de determinaciones A, no está incluida en el sistema, de modo que este la precediera y le atribuyera esa determinación temporal entre otras; sin asumir para cierto punto de vista el carácter de presente, no podemos llegar siquiera a construir una «serie A».

\section{De LA SITUACIONALIDAD A LA PLURALIDAD}

Ahora bien, aunque el núcleo de la solución merleaupontyana a la paradoja de McTaggart que hemos elaborado sea de hecho el que acabamos de resumir, es necesario sin embargo explorar algunas consecuencias polémicas de esta solución, si es que pretendemos que ella pueda sostenerse. En concreto, tendremos que referirnos al problema de la pluralidad irreductible de descripciones de la realidad temporal, para lo cual recurriremos a dos vías. A lo que queremos llegar por estos dos caminos es a establecer que un acercamiento situacional al problema del tiempo tiene que ser ipso facto un acercamiento «pluralista» al mismo, en el sentido de que -como veremos a partir de ambas comparaciones- plantear que el tiempo solo puede ser conocido desde algún punto de vista conduce inmediatamente a establecer que la realidad temporal debe ser conocida desde una pluralidad, irreductible, de puntos de vista; que adoptar un abordaje situacional significa que ya no podremos describir la realidad de un único modo.

La primera puesta en paralelo que queríamos realizar, pues, es un cruce con un texto de Michael Dummett, un clásico en la discusión anglosajona, que, quince años después de la $P h P$, aborda la paradoja de McTaggart sobre la base de premisas muy similares a las que hemos reconstruido desde el ajuste de cuentas merleaupontyano con la filosofía trascendental. Para Dummett, cuando McTaggart argumenta en torno a la superposición inconsistente de las «determinaciones A» de presente, pasado y futuro que -según vimos- nos obliga a embarcarnos en una búsqueda infinita de puntos de referencia, puesto que ningún momento posee en términos absolutos ninguno de estos tres caracteres, 
lo que se está demostrando no es en rigor la irrealidad del tiempo, sino que podemos llegar a esa conclusión solo si compartimos una premisa ontológica más general: la idea de que la realidad tiene que poder ser descrita en los términos de una «descripción completa», que incluya el conjunto de los fenómenos sin apelar a ningún punto de vista (cfr. Dummett (1960): 503; Dummett (1978): 356). Según Dummett, precisamente lo característico del tiempo es que desafía esta pretensión de completitud: solo podemos tener la experiencia de una sucesión de eventos si podemos establecer cuál de ellos es el que estamos percibiendo ahora, y cuáles por el contrario son pasados o futuros; una subjetividad cognoscente que percibiera la totalidad de los eventos sin desplazarse a través de ellos sería incapaz de experimentar el tiempo, del mismo modo que un observador que percibiera una ruta pero no nuestro movimiento a lo largo de ella (cfr. Dummett (1960): 502; Dummett (1978): 355). Pero si, para recuperar la experiencia del tiempo, reponemos la percepción en sucesión de los distintos eventos, entonces nuevamente la descripción de la experiencia tendrá que señalar cada vez cuál es el evento actual, y con esto tendremos, naturalmente, distintas descripciones, con distintos caracteres temporales, de los mismos eventos. En términos de Dummett, en lugar de una descripción completa de la realidad solo podremos tener una variedad de descripciones máximas: una en la cual un cierto evento $\mathrm{M}$ sea pasado, otra en la cual sea presente, otra en la cual sea futuro, y ninguna de estas en particular será la realidad (incluso si, claro está, cada descripción se refiere al mismo conjunto de eventos, solo que variando su «ángulo» temporal; $c f r$. Dummett (1960): 503; Dummett (1978): 356). Solo podemos concebir la totalidad de la realidad temporal como suma de estas descripciones máximas, todas ellas situadas, y no desde la supresión de nuestra situacionalidad. Manteniendo esta última, por lo demás, resulta claro por qué la paradoja de McTaggart no puede surgir: dentro de esta pluralidad de descripciones situadas, no habrá ninguna descripción en la cual las determinaciones de presente, pasado y futuro se superpongan, que es un resultado al que se arribaba sobre la base de, precisamente, brindar una descripción no situada.

Pues bien, en la concepción merleaupontyana, que según hemos visto es también situacional -y que sobre esa base podía proveernos una solución a la paradoja de McTaggart-, ¿desemboca también esto en un tipo de pluralismo irreductible con respecto a las descripciones del tiempo? Aquí es donde introducimos nuestra segunda puesta en paralelo, ya no entre Merleau-Ponty y otro autor, sino esta vez interna a la propia $P h P$. En efecto, hablando sobre la perspectividad espacial, el filósofo francés establece una tesis «pluralista» muy similar a la de Dummett sobre el tiempo, y que, a la luz de otros pasajes, podríamos extrapolar al tiempo también para el caso del fenomenólogo. Nos referimos al texto del «Preámbulo» a la Primera Parte. Si, como se plantea 
para el ejemplo de una casa, ella «de sí no es ninguna» de las apariciones unilaterales que presenta a cada una de nuestras perspectivas situadas, ello no puede significar sin embargo que la casa misma sea tal como es vista «desde ninguna parte»; ver es siempre, nos recuerda Merleau-Ponty, ver desde algún lado (cfr. Merleau-Ponty (1985): 87). En consecuencia, corrige la formulación inicial y señala que si la casa misma no se reduce a ninguna perspectiva particular es porque se trata de «la casa vista desde todas partes» (ibid: 88). En otras palabras: la única forma en la que podemos superar la unilateralidad de cada una de nuestras percepciones situadas es, no una supresión de nuestra perspectividad, pretendiendo avanzar a un conocimiento sin punto de vista, sino la multiplicación de tales perspectivas; cada objeto espacial tendrá que pensarse como la suma ideal de una infinidad de perspectivas. Como ha insistido Bimbenet a este respecto, se trata de distinguir entre «un punto de vista que se intercambia lateralmente con otros [...] posibles, y el sueño intelectualista de una mirada milagrosamente liberada de su anclaje en un punto de vista. [...] Llegamos al mundo por la multiplicación de los puntos de vista, no por su abolición» (Bimbenet (2008): 103. Subrayado nuestro).

Como vemos, el análisis merleaupontyano de espacio hace una clara transición desde la situacionalidad de nuestra percepción a la irreductible pluralidad de las descripciones que tendremos que hacer de la realidad. Nuevamente en palabras de Bimbenet: «yo veo la cosa desde aquí, pero [...] podría también verla de allí, de allí y de allí, ad libitum. Ella es el objeto de una pluralidad de miradas posibles [...] o de una multiplicidad perspectiva» (ibíd). Lo que nos interesa para nuestro tema, sin embargo, es el grado en que podamos extender la vinculación situacionalidad-«pluralismo» del caso del espacio al del tiempo. Si esta extrapolación «interna» a la obra de Merleau-Ponty fuera completa, lo cual asimismo significaría que podemos hacer la extrapolación «externa» desde el paralelismo con Dummett, hallaríamos que, así como la descripción de la totalidad de la realidad espacial consiste en una suma de miradas desde posibles «aquí», la descripción de la totalidad de la realidad temporal resulta de adicionar, también en pie de igualdad, la descripción del mundo tal como lo percibimos ahora junto con otras infinitas descripciones posibles; no es más real nuestro mundo actual que el que existió, digamos, hace cien años. Y tal acercamiento espacio-tiempo resulta, por lo demás, enteramente razonable: Merleau-Ponty no podría decir nunca que el conjunto de lo real es el mundo tal como sería percibido desde ningún tiempo, puesto que, como vimos al comienzo mismo de la exposición, esto nos haría recaer en la indeterminación temporal propia del trascendentalismo y (añadimos nosotros) de McTaggart. No tendríamos tiempo si lo observáramos como un objeto, si no tuviéramos un punto de vista en él; tal punto de vista es fundante de las distinciones inherentes al tiempo. Pero ese punto de vista no puede ser uno solo, no habrá 
habido siempre un único presente, o el tiempo estaría paralizado. Entonces, si la realidad no es tal como se la ve desde ningún momento, tendrá que ser como se la vea desde todos los momentos. E incluso, aunque este pluralismo no aparezca enunciado explícitamente en el capítulo «La temporalidad», podemos encontrar nuevamente en el preámbulo a la primera parte un pasaje que parece decir exactamente lo que estamos buscando: que «el objeto se ve [...] desde todos los tiempos igual a como se ve desde todas partes y por el mismo medio, la estructura de horizonte» (Merleau-Ponty (1985): 89).

Ahora bien, si no vamos a hacernos las cosas ilegítimamente fáciles, tenemos que analizar un poco mejor qué quiere decir este pasaje. Un paralelismo exacto con el espacio significaría que si en cierto momento nos pidieran una descripción de la totalidad de la realidad temporal, tendríamos que incluir en ella en pie de igualdad el mundo tal como es visto desde ese momento, digamos el año 2013, y también tal como es visto desde un «ahora» anterior, digamos el 2012. Esto parecería encontrar base textual cuando, siguiendo con el ejemplo de la casa, Merleau-Ponty insiste en que «aun cuando mañana se derrumbara, seguirá siendo verdad para siempre jamás que la casa existió hoy» (ibíd.); esto es, el presente no es más real que el pasado, no lo refuta como una tesis verdadera a una falsa, y el pasado sigue siendo parte del totum de la realidad espaciotemporal. No obstante, no es claro que el fenomenólogo esté pensando, en el preámbulo a la primera parte, en una equiparación "pluralista» de las distintas perspectivas temporales, la presente y las pasadas. Más que en una equivalencia irreductible entre estas perspectivas, la cual no daría al presente ningún privilegio, Merleau-Ponty parece estar pensando por el contrario en cómo cada presente «recobra» sin pérdida todo el pasado, lo reintegra en sí mismo, y en este sentido tiene una primacía sobre él; la descripción correcta de la totalidad del tiempo bien podría ser, en consecuencia, la que hacemos desde el presente. Retomemos el preámbulo a la primera parte:

Es indudable que [veo la casa] desde un cierto punto de mi duración, pero es la misma casa que vi ayer, un día más vieja [...]. Sí, también ella tiene su edad y sus cambios, pero, aun cuando mañana se derrumbara, seguirá siendo verdad para siempre jamás que la casa existió hoy; cada momento del tiempo toma a los demás como testigos [...]; cada presente hunde definitivamente un punto del tiempo que solicita el reconocimiento de todos los demás [...]. El presente guarda aún en sus manos el pasado inmediato, sin plantearlo en cuanto objeto, y tal como éste guarda de la misma manera el pasado inmediato que le precediera, el tiempo transcurrido es enteramente recogido y captado en el presente (ibid. Subrayado nuestro).

Reconocer, pues, la existencia pasada de la casa que se derrumbó, no reducir lo real a la perspectiva presente, no requiere colocar en pie de igualdad 
presente y pasado, como tendríamos que colocar en pie de igualdad dos «aquí»» distintos, porque existe para el tiempo un fenómeno de «recobramiento», de «resguardo» del pasado -en virtud del horizonte de retenciones-, que no tiene paralelo en el fenómeno de la espacialidad.

Sin embargo, subsiste todavía, pese a estas líneas, un sentido claro en que podemos hablar de la concepción situacional merleaupontyana, igual que de la de Dummett, como un abordaje pluralista sobre la realidad temporal. El motivo, en rigor, es bastante obvio: incluso si en un momento -por ejemplo el actual, el año 2013-, existe una descripción privilegiada de lo real, la dada desde el presente, la cual sobrepuja las pretensiones de las descripciones que podían formularse desde «ahoras» pasados, sin embargo forma parte de la misma dinámica de análisis el hecho de que la descripción actual será desplazada por una descripción futura, de modo que el pluralismo, a su modo, continuará siendo inevitable. No porque en cada momento deba tener cabida la pluralidad de descripciones anteriores, sino simplemente porque a este momento le sucederá otro, al que corresponderá su propia descripción de la realidad, y en consecuencia no podrá aspirar a una imagen definitiva de la realidad temporal. Si, en una descripción que demos ahora, el año 2013 es el presente, naturalmente, como teóricos que aceptamos las «determinaciones A», tendremos que admitir también una próxima descripción, en la que el mismo año figurará como pasado.

Recapitulando, pues, los resultados de esta segunda sección, podemos confirmar que el análisis merleaupontyano, al igual que el de Dummett, realiza un tratamiento de las «determinaciones A» que supone una pluralidad de descripciones de lo real, y por esta vía logra preservar tales determinaciones sin caer en algún tipo de inconsistencia como la denunciada por McTaggart y, tras él, por los distintos «teóricos B». Hemos pasado, pues, de la problemática de la situacionalidad a la de la pluralidad en la descripción de la realidad espaciotemporal. Ahora bien, ¿es este «momento pluralista» del análisis la última palabra de la cuestión del tiempo? ¿Es tan simple renunciar a la idea de una unificación de las distintas descripciones de la realidad? Las tesis de Smith, justamente, nos obligarán a preguntarnos esto.

\section{DE LA PLURALIDAD A LA COMPOSIBILIDAD}

En un trabajo del año 2011, Smith se introduce en el debate precisamente en este punto, cuestionando que pueda escaparse a la inconsistencia propia de las determinaciones A por la vía de atribuir distintas determinaciones a un mismo momento en descripciones diferentes. Es cierto, reconoce Smith, que -como señalamos respecto a Dummett- de este modo ninguna de las múltiples descripciones involucra una inconsistencia en las predicaciones de determinaciones A; la incompatibilidad se produciría únicamente entre descripciones 
distintas. Pero aquí entra en juego el supuesto clave, que el trabajo de Smith -si bien descansa en última instancia en un error- tiene el mérito de identificar: ¿por qué sería legítimo que estas distintas descripciones -por ejemplo, aquella en que 1800 es presente, y otra en que es pasado- se mantengan separadas? En palabras de Smith, los «teóricos A», esto es, aquellos que defienden la inclusión de las determinaciones de presente, pasado y futuro al reconstruir el tiempo, «querrán dibujar diferentes imágenes de la realidad en diferentes momentos». Pero, continúa,

estos diferentes diagramas [...] deben ser susceptibles de combinarse en un diagrama espaciotemporal tetradimensional. El teórico A no puede dibujar un diagrama que muestre la situación de 1800 , y otro que muestre la situación de $1900[\ldots]$ y luego sostener que los dos diagramas no pueden combinarse en uno [...] (Smith (2011): 241. Subrayado en el original).

Partimos, dice Smith, de atribuir a los «teóricos A» el supuesto muy básico de que existe una serie temporal única, esto es, de que todos los hechos temporales pueden estar incluidos en cada descripción, simplemente variando sus «determinaciones A», porque no hay hechos fuera de un único marco espaciotemporal. La situación no es asimilable a una en la cual existiera un «hipertiempo», de manera tal que, por ejemplo, en un hiper- $t_{1}, 1800$ fuera presente, $\mathrm{y}$ en un hiper- $\mathrm{t}_{2}$, el presente fuera 1900, y 1800 fuera pasado. En ese caso, las determinaciones incompatibles «ser 1800 presente» (desde el punto de 1800) y «ser 1800 pasado» (desde el de 1900) no pertenecerían por igual a nuestra serie temporal, y entonces sería legítimo no integrarlas en un único esquema temporal total. Cada uno de los presentes, y con él la determinación de otros momentos como pasados o futuros, sería interno a un hipertiempo; hiper- $t_{1}$ sería aquel en que 1800 es presente y 1900 futuro, e hiper- $t_{2}$ aquel en que 1900 es presente y 1800 pasado. Pero, reiteremos, esta formulación extravagante seguramente no es lo que tiene en mente el «teórico A», para quien más bien, simplemente, 1800 es presente en 1800 y es pasado en 1900, no en hipertiempos distintos. Demos nuevamente la palabra a Smith:

El teórico A [...] dice que el tiempo 1 -el tiempo en el que dibujó su primera imagen- fue [...] 1800. Pues bien, esa imagen puede ser incorporada a nuestro diagrama espaciotemporal único, porque todos los momentos en la imagen, $y$ el momento en el que la imagen fue dibujada, están en ese diagrama único (ibíd).

Esto es, por hipótesis, todos los momentos en juego en esta primera imagen pertenecen a la misma serie temporal. «Y de manera similar», continúa Smith, «para todas las otras imágenes». «Pero entonces», argumenta Smith para devolvernos a la paradoja, 
cuando sí combinamos las imágenes en un diagrama espaciotemporal único, instantáneamente vemos la contradicción en la teoría A: se debe mostrar que cada momento tiene las tres «propiedades A» incompatibles de presente, pasado y futuro (ibid).

Analicemos la argumentación de Smith. Su crítica es plausible en un sentido que quizá no se identifica a simple vista, a saber: ¿cómo podemos decir que distintos eventos pertenecen a la misma serie temporal y luego decir, sin embargo, que no podemos incluirlos en una única descripción? ¿En qué sentido sería ella, entonces, la misma, si no podemos superar su fragmentación? La paradoja de McTaggart reaparece, aquí, como consecuencia de un presupuesto en principio bastante inocente, y cuyas condiciones una serie de autores «pluralistas» en la filosofía del tiempo deberían poder satisfacer: el presupuesto de que el tiempo sea componible.

Pues bien, aunque Smith tiene razón en defender la composibilidad en contra de un «pluralismo radical», sin embargo su error radica en la forma que según él debería cobrar esta composibilidad. Smith, curiosamente, piensa que integrar las distintas perspectivas temporales situadas en una descripción única de la realidad temporal significa simplemente superponerlas. Si hacemos esto, que surja una inconsistencia es completamente predecible: dado que suprimimos en qué descripción de la realidad es que cada momento es presente, pasado o futuro, tendrá que tener los tres caracteres en la misma descripción. Pues bien, la respuesta que puede darse a esto sin caer en un «pluralismo radical» es, simplemente, que Smith carece de un equivalente de las nociones fenomenológicas de horizonte y de síntesis de transición.

En efecto, aunque hayamos identificado en la tematización merleaupontyana del tiempo un «momento» pluralista del análisis, que permitía poner al fenomenólogo en línea con Dummett, sin embargo no falta en los análisis merleaupontyanos, en absoluto, una preocupación por recobrar la unidad del tiempo. El problema, entonces, pasa por el tipo de síntesis que es necesario para que haya «el tiempo» y no solo «tiempos». Sabemos, ciertamente, que Merleau-Ponty rechaza una síntesis, una unificación, de los distintos momentos temporales que tuviera que cobrar la forma de un despliegue de todos ellos bajo la mirada omniabarcante de una conciencia constituyente. Pero el fenomenólogo opone a esto la noción husserliana de una síntesis de transición, cuya legitimidad para resolver el problema planteado por Smith quisiéramos remarcar. Delimitándose por igual de la solución trascendental y del tiempo «fragmentado» del empirismo, Merleau-Ponty se desliza de hablar de un sujeto que está en el tiempo a uno que es tiempo, esto es, a señalar que mientras el sujeto no puede realizar la síntesis unificadora de los momentos desde «arriba» o «fuera» del tiempo, tampoco puede estar «por debajo» o «dentro» del tiempo 
en el sentido de una intratemporalidad. La sucesión de los distintos presentes es, para Merleau-Ponty, un encadenamiento que se identifica con el despliegue de la subjetividad misma: no podemos unificar un flujo temporal más que, justamente, viviéndolo, «efectuando», gradualmente, la transición de un presente a otro antes que «contemplándola» como un proceso acabado. Unificar los distintos presentes no puede significar superponerlos unos a otros en una simultaneidad, sino precisamente experimentarlos como parte del mismo flujo, dentro del cual cada perspectiva desemboca en la siguiente:

el encadenamiento continuo de los campos de presencia [...] tiene como carácter esencial el no efectuarse más que paulatina y progresivamente; cada presente, por su misma esencia de presente, excluye la yuxtaposición con los demás presentes e, incluso en el pasado lejano, no puede abarcar cierta duración de mi vida más que desenvolviéndola de nuevo según su propio tempo. [...] Una vez más, la «síntesis» del tiempo es una síntesis de transición, es el movimiento de una vida que se despliega, y no hay otra manera de efectuarla que vivir esta vida [...] (Merleau-Ponty: 1985: 430-431).

Es en virtud de esto mismo, por otra parte, que no se ve cómo podría objetarse que la síntesis de transición sea, efectivamente, una síntesis. La unidad del tiempo no se ve atacada por la circunstancia de que solo pueda experimentársela «desde dentro», desde un presente, y esto precisamente por un punto que la reconstrucción del «pluralismo» temporal por parte de Smith -aunque también por un defensor de tal pluralismo, como el propio Dummett- soslaya por completo: las perspectivas temporales múltiples no son por ello perspectivas discretas; cada presente está rodeado por sus horizontes de pasado y futuro que permiten el encadenamiento de los momentos, y que implican, por otra parte, que separar una perspectiva de otras requiera de un acto de abstracción. Volviendo al problema de las «determinaciones A»: la perspectiva en la que $\mathrm{M}$ es un momento presente es otra, ciertamente, que aquella en la que ha cobrado el carácter de pasado, pero esta forma de pluralidad no introduce ninguna discontinuidad entre las experiencias del tiempo, precisamente en tanto $\mathrm{M}$ se ha conservado en el modo de la retención.

Recapitulando, pues:

1. El abordaje «situacional» merleaupontyano sobre el tiempo -y por lo tanto la solución que a partir de aquel podamos dar a la paradoja de McTaggart- efectivamente compromete al filósofo francés con una tesis «pluralista» análoga a la defendido por Dummett -y que el fenomenólogo no explicita con igual claridad respecto al tiempo que con relación al espacio-.

2. No obstante, este pluralismo no es más que, podemos decir, un «momento» de la exposción merleaupontyana, y no supone el compromiso con algún 
tipo de pluralismo «radical» que hiciera imposible el «momento», teóricamente posterior, de la síntesis. A diferencia del caso de Dummett, Merleau-Ponty sí aborda el problema de la composibilidad de las diferentes descripciones de la realidad temporal. Y, por esta vía, no se hace susceptible de las recientes críticas de Smith contra las teorías que defienden la consistencia de los caracteres de presente, pasado y futuro.

\section{REFERENCIAS BIBLIOGRÁFICAS}

Bimbenet, Étienne, «Un motif d'étonnement majeur: le perspectivisme», en Alter. Révue de phénoménologie, No. 16, 2008.

Cormick, Claudio, «Tiempo y situacionalidad: la 'respuesta' de Merleau-Ponty a la paradoja de McTaggart», Ideas y valores, Bogotá, Colombia, No 156, 2014 (en prensa).

Dummett, Michael, «A Defense of McTaggart's Proof of the Unreality of Time», en The Philosophical Review, Vol. 69, No. 4, Oct., 1960.

Dummett, Michael, Truth and other enigmas, Harvard University Press, Cambridge, Massachussets, 1978.

McTaggart, J. M. E., «The Unreality of Time», en Mind, Vol. 17, No. 68, Oct., 1908.

McTaggart, J. M. E., The nature of existence, vol. II, Cambridge, Cambridge University Press, 1927.

Merleau-Ponty, Maurice, Fenomenología de la percepción, Barcelona, PlanetaAgostini, 1985.

Merleau-Ponty, Maurice, Lo visible y lo invisible, seguido de: Notas de trabajo, Buenos Aires, Nueva Visión, 2010.

Sмith, N., «Inconsistency in the A-Theory», Philosophical Studies: an international journal for philosophy in the analytic tradition, 156(2), 2011.

Claudio Cormick becario doctoral del Consejo Nacional de Investigacones Científica y Técnicas (CONICET). Doctorando en la Universidad de Buenos Aires (UBA) eco-tutela con Université Paris VIII (Vincennes-Saint-Denis).

Publicaciones recientes: «Tiempo y situacionalidad: la 'respuesta' de Merleau-Ponty a la paradoja de McTaggart», Ideas y valores, Bogotá, Colombia, Nº 156, diciembre de 2014 (en prensa); «Cómo recibir a Merleau-Ponty en la «theory of time». Una polémica», Tábano, Buenos Aires, 2014 (en prensa). 
Línea de investigación: actualmente el autor se centra en un cruce entre la fenomenología merleaupontyana del tiempo y los debates en la «theory of time» de la filosofía analítica, como parte de un proyecto más general acerca de la concepción «situacional» del tiempo.

Dirección postal: Tucumán 3775, $7^{\circ}$ A, Ciudad Autónoma de Buenos Aires, Argentina

Dirección electrónica: claudiocormick@yahoo.com.ar 